University of Nebraska - Lincoln

DigitalCommons@University of Nebraska - Lincoln

Faculty Publications, Department of Physics and Astronomy

Research Papers in Physics and Astronomy

2005

\title{
Electron Diffraction from Free-Standing, Metal-Coated Transmission Gratings
}

\author{
Glen Gronniger \\ University of Nebraska - Lincoln \\ Brett E. Barwick \\ University of Nebraska-Lincoln, brett.barwick@trincoll.edu \\ Herman Batelaan \\ University of Nebraska - Lincoln, hbatelaan@unl.edu \\ Tim Savas \\ Massachusetts Institute of Technology \\ David E. Pritchard \\ Massachusetts Institute of Technology, dpritch@mit.edu \\ See next page for additional authors
}

Follow this and additional works at: https://digitalcommons.unl.edu/physicsfacpub

Part of the Physics Commons

\footnotetext{
Gronniger, Glen; Barwick, Brett E.; Batelaan, Herman; Savas, Tim; Pritchard, David E.; and Cronin, Alex, "Electron Diffraction from Free-Standing, Metal-Coated Transmission Gratings" (2005). Faculty Publications, Department of Physics and Astronomy. 117.

https://digitalcommons.unl.edu/physicsfacpub/117
}

This Article is brought to you for free and open access by the Research Papers in Physics and Astronomy at DigitalCommons@University of Nebraska - Lincoln. It has been accepted for inclusion in Faculty Publications, Department of Physics and Astronomy by an authorized administrator of DigitalCommons@University of Nebraska Lincoln. 


\section{Authors}

Glen Gronniger, Brett E. Barwick, Herman Batelaan, Tim Savas, David E. Pritchard, and Alex Cronin 


\title{
Electron diffraction from free-standing, metal-coated transmission gratings
}

\author{
Glen Gronniger, Brett Barwick, and Herman Batelaan ${ }^{a)}$ \\ Department of Physics and Astronomy, University of Nebraska-Lincoln, 116 Brace Laboratory, Lincoln, \\ Nebraska 68588-0111 \\ Tim Savas and Dave Pritchard \\ Research Laboratory of Electronics, Massachusetts Institute of Technology, Cambridge, \\ Massachusetts 02139-4307
}

Alex Cronin

Department of Physics, University of Arizona, Tucson, Arizona 85721

(Received 5 May 2005; accepted 1 August 2005; published online 14 September 2005)

\begin{abstract}
Electron diffraction from a free-standing nanofabricated transmission grating was demonstrated, with energies ranging from $125 \mathrm{eV}$ to $25 \mathrm{keV}$. Observation of 21 diffraction orders highlights the quality of the gratings. The image charge potential due to one electron was measured by rotating the grating. These gratings may pave the way to low-energy electron interferometry. (C) 2005 American Institute of Physics. [DOI: 10.1063/1.2053347]
\end{abstract}

Pioneering work in atom optics ${ }^{1}$ led to the development of $100-\mathrm{nm}$ period silicon nitride transmission gratings with free-standing bars. ${ }^{2}$ Such gratings have since been used for $\mathrm{Na}$ atom interferometry, ${ }^{3} \mathrm{He}_{2}$ molecule diffraction ${ }^{4}$ and Bucky ball diffraction. ${ }^{5}$ In this Letter we report that freestanding gratings can also diffract low-energy electron beams. This provides a high throughput alternative to the use of bi-prisms. ${ }^{6}$ Interactions between electron de Broglie waves and the grating structure do affect the diffraction patterns. Our analysis suggests that Mach-Zehnder ${ }^{3}$ and Talbot-vonLau ${ }^{7}$ interferometry using these gratings is now possible with low energy electrons. This opens new possibilities for electron physics as indicated in the conclusion.

Until now, transmission diffraction of low-energy electrons has been problematic. For example, solid crystals that have been used for interferometry ${ }^{8,9}$ absorb low energy electrons. Thin collodion films coated with gold stripes were developed, ${ }^{10}$ but these also suffer from low transmission. In collodion, $125 \mathrm{eV}$ electrons undergo inelastic scattering in a distance much less than $10 \mathrm{~nm} .{ }^{11}$ Free standing transmission gratings avoid this problem because the physical channels between the bars transmit low energy electrons without inelastic scattering. Free-standing gratings were pioneered by Jönsson, ${ }^{12}$ but their regularity was poor. Now, high transmission combined with good quality diffraction of low-energy electrons, Fig. 1, has been achieved for the first time by using metallized silicon nitride nanostructures with $50-\mathrm{nm}$ channels between $50 \mathrm{~nm}$ wide free-standing bars, Fig. 2.

We have diffracted electrons with kinetic energy ranging from $125 \mathrm{eV}$ to $25 \mathrm{keV}$ which corresponds to de Broglie wavelengths, $\lambda_{\mathrm{dB}}$, ranging from 110 to $7.8 \mathrm{pm}$. Electron beams for this study have been generated by two methods: first, in a dedicated beam apparatus described below, and second, with the converging beam of an electron microscope described later. In each case the total transmission is about $30 \%$, which is certainly high enough to envision future interferometer experiments using multiple gratings. A major challenge for this plan is to understand the effect of image

\footnotetext{
${ }^{\text {a) }}$ Author to whom correspondence should be addressed; electronic mail:
} hbatelaan2@unl.edu charge potentials and any random potentials that may cause decoherence. An analysis including these potentials is discussed after the data presented below.

Our diffraction experiment is straightforward: electrons were emitted from a thermionic source at energies, $E$, ranging from 125 to $900 \mathrm{eV}$, with an energy width, $\Delta E$, of about $1 \mathrm{eV}$. This electron beam was collimated by a $5 \mu \mathrm{m}$-wide molybdenum slit, followed $25 \mathrm{~cm}$ downstream by a $2 \mu \mathrm{m}$ wide by $10 \mu \mathrm{m}$ tall slit that was made using a focused ion beam to mill a $100 \mathrm{~nm}$ thick silicon nitride substrate. The second slit was coated first with titanium and then with gold on both sides. The angular spread of electron trajectories that can travel straight through the collimation slits is $\theta_{\text {geom }}=(5$ +2) $\mu \mathrm{m} / 25 \mathrm{~cm}=2.8 \times 10^{-5} \mathrm{rad}$. This agrees with the observed angular spread for our $500 \mathrm{eV}$ electron beam which produces a $7 \mu \mathrm{m}$ wide spot (FWHM) in the plane of detection, located $31 \mathrm{~cm}$ downstream of the $2 \mu \mathrm{m}$ slit. The collimation is much better than the diffraction angle, $\theta_{\text {diff. At }}$. the second slit the beam has a transverse coherence length of $l_{t}$ $=0.89 \mathrm{~h} / \Delta p=2 \mu \mathrm{m}^{13}$ which means that the beam is nearly diffraction limited. The roughly $125 \mathrm{~nm}$ thick silicon nitride grating (Fig. 2) was placed $7 \mathrm{~cm}$ downstream from the second collimation slit. We infer from this geometry that the electron spot size on the grating is approximately $4 \mu \mathrm{m}$, and

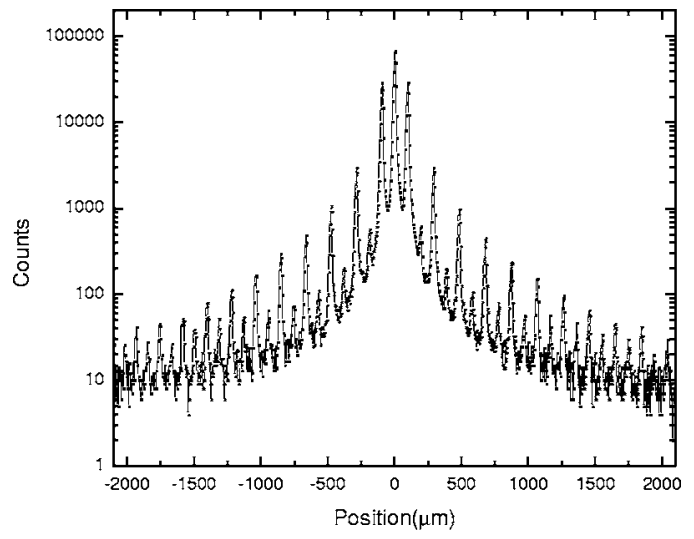

FIG. 1. Electron beam diffraction with an electron energy of $900 \mathrm{eV}$. Positive and negative orders are resolved out to the 21 st order. 


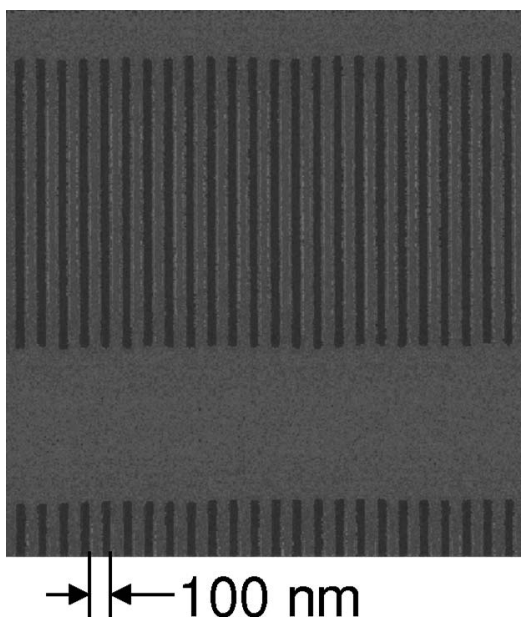

FIG. 2. Nano-fabricated 100-nm period grating.

coherent over (at least) $2 \mu \mathrm{m}$. This is consistent with the transverse coherence length inferred from the observed resolution, $R$, of the diffraction peaks. The resolution is defined as the ratio of the peak separation over the peak width and $d$ is the grating periodicity. The coherence length is given by $l_{t}=R d=(135 / 11) \times 100 \mathrm{~nm} \approx 1 \mu \mathrm{m}$ (at $500 \mathrm{eV}$, see Fig. 3). This means that the electron beam is nearly diffraction limited, and the grating does not affect the coherence length much at higher energies. Beam broadening due to the energy width of the electron beam, $\Delta \theta_{\text {diff }} / \theta_{\text {diff }}=n \Delta \lambda_{\mathrm{dB}} / \lambda_{\mathrm{dB}}$ $=n \Delta E / 2 E \approx n 10^{-3}$, where $n$ is the diffraction order, is negligible (the associated longitudinal coherence length and time are about $50 \mathrm{~nm}$ and $5 \times 10^{-15} \mathrm{~s}$ ). The grating had been metallized with a $2 \mathrm{~nm}$ layer consisting of $60 \%$ gold and $40 \%$ palladium. The detector is a channeltron placed behind another $5 \mu \mathrm{m}$ molybdenum slit. By controlling the thermionic source current, we limit the count rates to $10^{6}$ electrons per second to protect the channeltron. Combined with our electron velocity of $10^{7} \mathrm{~m} / \mathrm{s}$ this means that only one electron is in our system at any one time, which excludes the possibility of any electron-electron interaction. The apparatus is operated in a $10^{-7}$ Torr turbo pumped vacuum system and is shielded from the earth's magnetic field by two layers of magnetic shielding. The detected far field diffraction patterns are shown in Fig. 3. Figures 3(a) and 3(b) are taken at 500 and $125 \mathrm{eV}$, respectively. They are taken by sweeping the diffraction pattern across the detection slit using deflection plates, while electron counts were collected with a multi channel scaler board. No background has been subtracted.

In a separate experiment, a similar metal coated grating was placed in an Hitachi S-2460N scanning electron microscope (SEM) after the objective lens. The SEM pictures show evidence of electron beam diffraction for energies ranging from $500 \mathrm{eV}$ to $25 \mathrm{keV}$. This demonstrates that the grating can be used in conjunction with existing electron microscope technology, and can tolerate a pA of current spread over a $30 \mu \mathrm{m}$ diam spot. We failed to observe a diffraction pattern from the unmetallized silicon nitride gratings in either apparatus, presumably because of surface charge.

A path integral calculation without any interaction between the electron and the grating does not agree well with the data, especially at the lower energies. Neither the peak widths nor the relative peak heights agree well. Only the peak positions are matched. However, a modified analysis

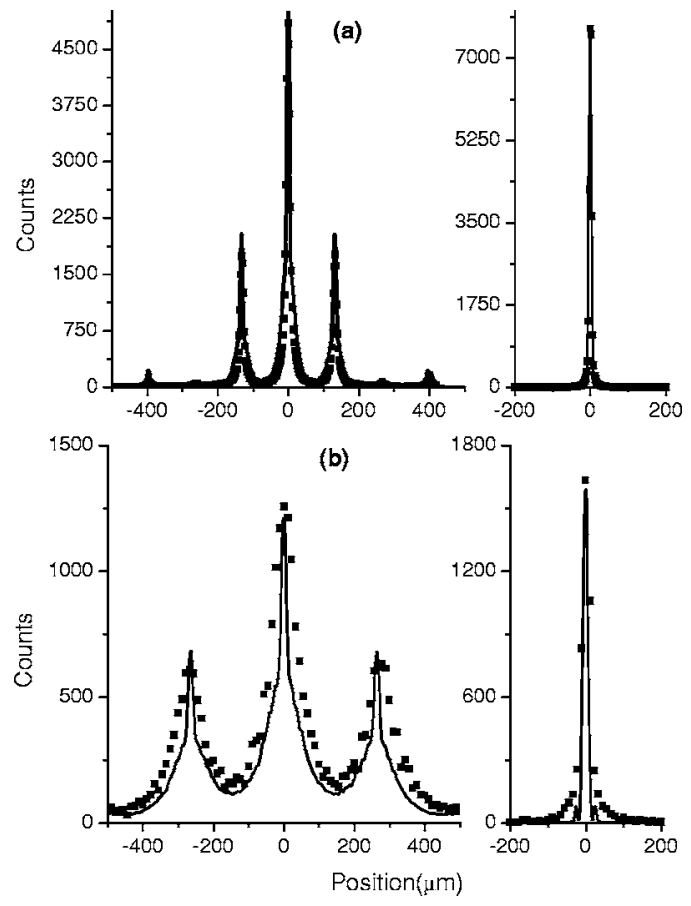

FIG. 3. Electron beam diffraction. The electron diffraction is presented as a function of position for electron energies of (a) $500 \mathrm{eV}$ and (b) $125 \mathrm{eV}$. The beam profile without a diffraction grating is shown for comparison. The result of a path integral calculation is represented by the solid line.

that includes a spatially dependent potential, $V(x)$ $=V_{\text {image }}(x)+V_{\text {random }}(x)$ ( $x$ is perpendicular to beam axis and grating bars), to describe the interaction between the electron and the grating channel walls, Eq. (1), does improve the agreement to the diffraction data, Fig. 3. One potential is based on the method of images for an electron next to a conducting wall, Eq. (2), and an additional random potential, Eq. (3), that appears to be needed to explain the broadening of the diffraction peaks,

$$
\begin{aligned}
& \psi=N \sum_{\text {path }} e^{i \varphi_{\text {path }}} ; \quad \varphi_{\text {path }}=\frac{L_{\text {path }}}{\lambda_{\mathrm{dB}}} 2 \pi-\frac{q V(x) t}{\hbar}, \\
& V_{\text {image }}(x)=\frac{q_{\text {image }}}{4 \pi \epsilon_{0}(w+2 x)}+\frac{q_{\text {image }}}{4 \pi \epsilon_{0}(w-2 x)} .
\end{aligned}
$$

A free parameter in Eq. (2) is $q_{\text {image }}$ and $q=-e$ in Eq. (1). The total width of the channel between the two grating bar walls is denoted by $w$. For the curve in Fig. 3 the value of $q_{\text {image }}$ that worked best was $e / 3.6$.

The random potential is given by

$$
V_{\text {random }}(x)=\sum_{i} A_{i} e^{-4 \ln 2\left(x-x_{i}\right)^{2} / \sigma_{i}^{2}}
$$

The use of the random potential was motivated by the irregularity of the crystalline structure on the grating surface. ${ }^{14}$ The detailed shape of the random potential (other than the typical length scale and strength) is not critical. A series of Gaussians was chosen for simplicity. In Eq. (3), $A_{i}$ and $\sigma_{i}$ are obtained from random number generators. The random numbers $A_{i}$ and $\sigma_{i}$ are normally distributed with means of $0 \mathrm{eV}$ and $250 \mathrm{~nm}$ and FWHM of $0.4 \mathrm{eV}$ and $250 \mathrm{~nm}$, respectively. The set $\left\{x_{i}\right\}$ of centers is regularly spaced and separated by $2 \sigma_{i}$. This potential has a characteristic lateral structure of 250 $\mathrm{nm}$. Incoherent averaging of multiple diffraction patterns for 


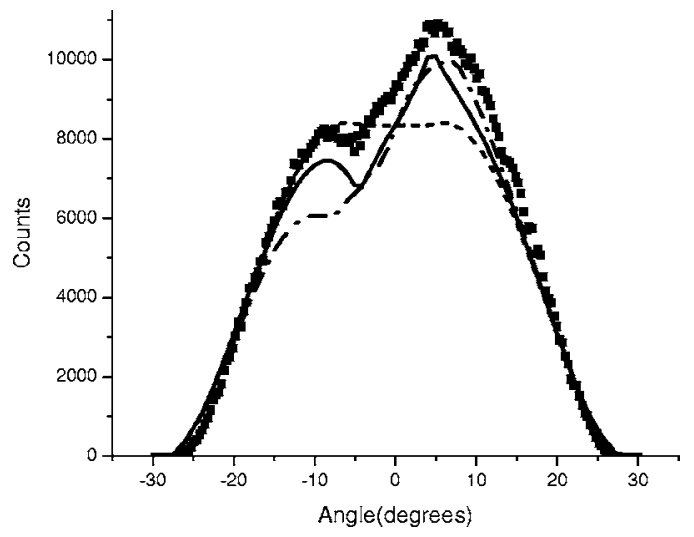

FIG. 4. Rocking curve for $500 \mathrm{eV}$. Electron transmission in the 1st order is given as a function of grating angle. Results are given for diffraction theory with no electron grating interaction (dashed line), with image charge potential (dash dot line), and with reduced image charge potential (solid line).

different random potentials effectively reduces the transverse coherence length to approximately $250 \mathrm{~nm}$ and, hence, broadens the transmitted beam and diffraction peaks.

The broadening of the diffraction peaks, Fig. 3(b), suggests a decoherence mechanism that may reduce interference fringe contrast. For one of the worst case scenarios we estimated this effect for interferometry. An interferometer may use a second grating to recombine two diffracted beams. The two diffracted beams pass through different parts of the second grating and thus accumulate a different spatially varying phase. Our model based on random potentials allows us to predict the resulting interferometric contrast when these two beams are once again overlapped. Using a detection slit of $150 \mu \mathrm{m}$ we find a contrast of $27 \%$ and $15 \%$ for 500 and 125 $\mathrm{eV}$, respectively, while for a detection slit of $10 \mu \mathrm{m}$ we find a contrast of $64 \%$ and $35 \%$ for 500 and $125 \mathrm{eV}$. So there is encouraging evidence that low energy electron interferometry is feasible with these gratings.

In Fig. 4 the electron transmission into the first diffraction order is given (square dots) as a function of the grating angle. This permits a measurement of the image charge potential. The dashed line is the result of diffraction theory with no interaction between the electron and the grating bars. The dash dot line is the result of the same calculation with a potential added that describes the image charge interaction between a static electron and two infinite walls, Eq. (2). ${ }^{15}$ The solid line is the same calculation with the image potential reduced by a factor of 3.6 in strength. The asymmetry in the model is due to the cross-sectional geometry of the grating bars combined with the image charge. ${ }^{16}$

In summary, free-standing nanostructure diffraction gratings have the advantage that they work for low energy electrons and we expect that such gratings can be used in new electron interferometry experiments, as well as other charged particle interferometers. ${ }^{17}$ The data shown in Fig. 3 were obtained with a diffraction-limited electron beam; however, much less collimation is required to observe diffraction, and even less collimation is needed for Talbot-vonLau (near field) interferometry. Hence, we think it is likely that nanostructured gratings can simultaneously play the role of the coherent source and beam splitter in electron interferometry. Applications for low-energy electron interferometers include measurements of the index of refraction due to forward scattering from atoms, ${ }^{18}$ sensing vector, ${ }^{19-22}$ and scalar potentials. $^{23}$

The authors thank Hank Smith for the production of the gratings in his laboratory. We thank Mike Chapman for helpful discussions. We also thank Sy-Hwang Liou for the use of his focused ion beam to make a collimation slit. This material is based upon work supported by the National Science Foundation under Grant No. 0112578, and by the Department of the Army under Grant No. DAAD1902-1-0280.

${ }^{1}$ D. W. Keith, M. L. Schattenburg, H. I. Smith, and D. Pritchard, Phys. Rev. Lett. 61, 1580 (1988).

${ }^{2}$ T. A. Savas, S. N. Shah, M. L. Schattenburg, J. M. Carter, and H. I. Smith, J. Vac. Sci. Technol. B 13, 2732 (1995).

${ }^{3}$ D. W. Keith, C. R. Ekstrom, Q. A. Turchette, and D. E. Pritchard, Phys. Rev. Lett. 66, 2693 (1991).

${ }^{4}$ W. Schollkopf and J. P. Toennies, J. Chem. Phys. 104, 3 (1996).

${ }^{5}$ M. Arndt, O. Nairz, J. V. Andrae, C. Keller, G. van der Zouw, and A. Zeilinger, Nature 401, 680 (1999).

${ }^{6}$ F. Hasselbach, Z. Phys. B: Condens. Matter 71, 443 (1988).

${ }^{7}$ J. F. Clauser and S. Li, Phys. Rev. A 49, R2213 (1994).

${ }^{8}$ L. Marton, J. A. Simpson, and J. A. Suddeth, Phys. Rev. 90, 490 (1953).

${ }^{9}$ L. Marton, J. A. Simpson, and J. A. Suddeth, Rev. Sci. Instrum. 25, 1099 (1954).

${ }^{10}$ V. P. Holl, Optik (Stuttgart) 30, 116 (1969).

${ }^{11}$ P. Pianetta, X-ray Data Booklet, Lawrence Berkeley National Laboratory, Sec. 3.2, low energy electron ranges in matter 2001, http://xdb.lbl.gov/

${ }^{12}$ C. Jönsson, Am. J. Phys. 42, 4 (1974).

${ }^{13}$ O. Nairz, M. Arndt, and A. Zeilinger, Phys. Rev. A 65, 032109 (2002).

${ }^{14}$ T. Schenkel, E. A. Stach, V. Radmilovic, S.-J. Park, and A. Persaud, Sample method for formation of nanometer scale holes in membranes (24 February 2003). Lawrence Berkeley National Laboratory. Paper LBNL52206, http://repositories.cdlib.org/lbnl/LBNL-52206

${ }^{15}$ D. K. Ross, Nuovo Cimento 110A, 571 (1997).

${ }^{16}$ A. E. Cronin and J. D. Perreault, Phys. Rev. A 70, 043607 (2004).

${ }^{17}$ F. Hasselbach and U. Maier, in Quantum Coherence and Decoherence: Proceedings ISQM-Tokyo '98, edited by Y. Y. Ono and K. Fujikawa (Elsevier, Amsterdam, 1999), pp. 299-302.

${ }^{18}$ R. C. Forrey, A. Dalgarno, and J. Schmiedmayer, Phys. Rev. A 59, R942 (1999).

${ }^{19}$ Y. Aharonov and D. Bohm, Phys. Rev. 115, 485 (1959).

${ }^{20}$ T. H. Boyer, Found. Phys. 32, 41 (2002).

${ }^{21}$ Y. Aharonov and T. Kaufherr, Phys. Rev. Lett. 92, 070404 (2004).

${ }^{22}$ Y. Aharonov, S. Popescu, B. Reznik, and A. Stern, Phys. Rev. Lett. 92, 020401 (2004).

${ }^{23}$ G. Matteucci and G. Pozzi, Phys. Rev. Lett. 54, 2469 (1985). 